\title{
Pulmonary Embolism in Patients with Chronic Lung Disease
}

\author{
Gilbert Berdine MD
}

Tompkins et al have written a scholarly examination of an important dilemma: the difficulty in suspecting a diagnosis of pulmonary embolism in patients with chronic dyspnea. ${ }^{1}$ The article has two parts, both of which make for worthwhile study. The article presents a concise discussion of the diagnosis and treatment of pulmonary embolism which even seasoned practitioners will find value in reading. The more important part of the article is a discussion of the difficulties facing a practitioner making a diagnosis of pulmonary embolism in patients with other causes for dyspnea.

Part of the dilemma is left largely unspoken: the diagnosis of COPD has morphed from a specific problem with specific inclusion and exclusion criteria to a general catch all term for anyone with dyspnea or cough who has ever been near a lit cigarette. Although the exact ICD9 (10) code may vary, they all get treated as if they had exacerbation of COPD.

The other part of the dilemma was explicitly discussed at length: in which patients who present with worsening respiratory symptoms should we suspect pulmonary embolism? I find myself in substantial agreement with the authors with one addition to suggest and two minor disagreements with their conclusions.

It is imperative that practitioners treat diagnoses as works in progress rather than divine writs. We should always have an image of a projected clinical

Corresponding author: Gilbert Berdine MD

Contact Information: Gilbert.Berdine@ttuhsc.edu

DOI: 10.12746/swrccc2014.0208.110 trajectory, and when the actual clinical picture deviates substantially from our projection, we must consider the possibility that our diagnosis is incorrect. The authors correctly point out that one should consider pulmonary embolism whenever we cannot find evidence for worsening airflow obstruction or some other credible pulmonary explanation for the worsening of symptoms. I would like to add to this thought: serial spirometry values are helpful. Whenever one of my COPD patients complain of worsening dyspnea, and the FEV1 is unchanged (or even improved) compared with baseline, and there are objective findings to match the subjective complaints, I consider other diagnoses with pulmonary embolism at the top of the list. In my own practice I have made the diagnosis of pulmonary embolism at the time of presentation a number of instances when these conditions presented themselves.

The other situation the authors discussed was the patient who fails to respond to treatment as expected. This is another variation on the theme of deviation from projected trajectory. Unfortunately, this is where the problem of conflating COPD with every respiratory ailment raises its ugly head. One can hardly expect patients without COPD to respond to treatment for COPD. The authors do not make this mistake, but others frequently do.

Even when the diagnosis of COPD is correct, not all patients with COPD respond rapidly to steroids. In a previous article in this journal, ${ }^{2}$ I suggested stages of COPD based on steroid responsiveness. If a patient has responded slowly (or not at all) to therapy in the past, one could hardly expect the patient to promptly improve with treatment. Should we obtain CT-PA in every hospitalization for one of these patients? I think not. This situation will require very astute clinical judgment to discern a substantial 
deviation from the patient's usual clinical trajectory.

Does COPD predispose to deep venous thrombosis (DVT)? The authors suggest that it does. My own experience says no. I think that the key element is whether the patient is ambulatory at the time of admission or not. In my own practice, if a patient with exacerbation of COPD is ambulatory at the time of admission, I do not employ prophylactic measures against DVT and I have never (30 years of practice) seen a single case of PE in these hospitalized patients. If, on the other hand, the patient is not ambulatory, then I always employ prophylactic measures against DVT. The ambulation (or lack thereof) criteria will be met for all ICU patients, patients who require non-invasive ventilator support, and many patients with hypoventilation syndromes.

I believe that the authors are correct in their suggestion that a correlation exists between obstructive sleep apnea (OSA) and DVT, but I suspect that this will be true only for the most severe cases where hypoventilation during wakefulness is present and it will be due to lack of ambulation. It would be an interesting study to see if OSA patients, properly controlled for ambulation, exhibit a risk for DVT.

My second point of contention is with the suggested utility of the D-dimer assay. I have no doubt that an experienced statistician can find some significant relationship between D-dimer and PE. Is this useful in practice? I think not. More important, reliance on D-dimer becomes a crutch which impedes the development of clinical judgment. It is clinical judgment that is the most reliable tool for suspecting PE in patients with suspected chronic pulmonary disease.

I offer a game analogy. Consider the two games of chess and go. Chess has been successfully reduced to an algorithm and computerized. Go has not. It is said that the best go players cannot even explain why they make the moves that they make. In my opinion, the problem of suspecting PE in patients with chronic pulmonary disease will be more like the game of go than the game of chess.
Author Affiliation: Gilbert Berdine is a pulmonary physician in the Department of Internal Medicine, Texas Tech Uiversity Health Science Center in Lubbock, TX.

Received: 09/22/2014

Accepted: 9/26/2014

Reviewers: Kenneth Nugent MD

Published electronically: 10/15/2014

Conflict of Interest Disclosures: None

\section{REFERENCES}

1. Tompkins RB, Harris V, Brown C, Griffith DE. Diagnosing Pulmonary Embolism in Patients with Suspected or Established Chronic Lung Disease. The Southwest Respiratory and Critical Care Chronicles. 2014; 2(8):5-16.

2. Berdine G. Clinical staging of COPD. Southwest Respiratory and Critical Care Chronicles 2013; 1(2): 44-45. 